J. Asiat. Soc. Bangladesh, Sci. 44(2): 149-158, December 2018

\title{
AN ANNOTATED AVIFAUNAL CHECKLIST OF THE SAINT MARTIN'S ISLAND OF BANGLADESH
}

\author{
IRIN SULTANA, SHAYER MAHMOOD IBNEY ALAM AND DELIP K. DAS* \\ Department of Zoology, Jagannath University, Dhaka-1100, Bangladesh
}

\begin{abstract}
Despite being an Ecologically Critical Area (ECA) and a key attraction place for its uniqueness and unique bird fauna to the bird watchers and researchers, a method-based, scientific checklist on the avifauna of the Saint Martin's Island is still lacking. This study was attempted to fill in this gap and produced an annotated bird checklist for the St. Martin's island. A total of 112 species of birds belonging to 36 families under 15 orders was listed. Of these, $37(33 \%)$ species were passerine and $75(67 \%)$ species were nonpasserine. Eleven $(9.82 \%)$ species were found to be very common, $12(10.72 \%)$ species common, nine $(8.04 \%)$ species uncommon and 80 (71.43\%) species rare.
\end{abstract}

Key words: Avifauna, Transect line, Shoreline, Saint Martin's Island, Checklist

\section{Introduction}

Birds are one of the key indicators that play an important role in the food web of an ecosystem (Zöckler 2005). In an ecosystem, birds represents almost all the functional groups that can help to keep all processes going and have the potentials to send important messages on the state of the environment (Zöckler 2005). As found in every ecosystem, birds are highly susceptible to any changes in the habitat they live in, and hence, are good biological indicators and are used in bioassays (Ahsan and Khanom 2005). Regular assessment of the avifaunal status of an ecosystem, therefore, can help to interpret the biological health of that ecosystem.

Bangladesh is a small country of 147,570 square $\mathrm{km}$ (Khan 2008). It has a rich biological heritage due to its geographical location between the two major biotic sub regions of the Oriental Region: The Indo-Himalayas and Indo-China (Khan 2008). A total of 138 mammalian species, 566 birds, 167 reptiles and 49 amphibians are reported to occur in Bangladesh (IUCN Bangladesh 2015). In addition, Bangladesh lies at the junction of the Central Asian and East Asian-Australasian flyways (routes that shorebirds use during their migration from the breeding to non-breeding grounds), offering key wintering and staging grounds to numerous migratory waders many of which are of international conservation concern (Chowdhury 2011).

*Author for correspondence: Email: bisharga1095@gmail.com 
The Saint Martin's Island is a unique habitat in Bangladesh because it is the only place where coral colonies are found. It has been recognized as an Ecologically Critical Area (ECA) by the Government of Bangladesh in 1999 (DoE 2015) and an important stepping stone along the migration route of many globally concerned migratory birds (BOBLME 2015). Rare bird like Pacific Reef Egret (Egretta sacra) and Rosy Starling (Pastor roseus)is also found here (Chowdhury 2016, The Daily Star 2010) and an attractive destination for bird watchers. Besides, all five species of marine turtle (including three globally threatened species) known to occur in Bangladesh have been reported from this area (Rashid and Islam 2005). St. Martin's Island is not only significant for its biodiversity value, but also important for Bangladesh in defining its exclusive economic zone and delineating its sea boundary in accordance with the United Nations Convention on the Law of the Sea (Thompson and Islam 2010). However, unregulated tourism, local population explosion and their unsustainable extraction of natural resources have become a great threat to the stability of this ecosystem (Thompson and Islam 2010).

Despite being an Ecologically Critical Area (ECA) and a key attraction place for its uniqueness and unique bird fauna to the bird watchers and researchers, a method-based, scientific checklist on the avifauna of the St. Martin's Island is still lacking. A checklist provides the background on the bird diversity of a place, the uniqueness of that place and to determine the importance of that place based on the presence of endemic, ecologically important or threatened bird species. Moreover, a checklist of any site also provides an accurate reference list and inventory for conservation workers, students, educators, ecologists and naturalists to facilitate documentation and recording of observations (Bryan et al. 2006). Therefore, this study attempted to produce an annotated bird checklist for the St. Martin's Island.

\section{Materials and Methods}

The Saint Martin's Island is the only coral-associated island of Bangladesh and located between latitude $20.567^{\circ}$ and $20.650^{\circ} \mathrm{N}$, and longitude $92.327^{\circ}$ and $92.327^{\circ} \mathrm{E}$ (Fig. 1). The total area of this island is only $8 \mathrm{~km}^{2}$ (3.1 sq. mile) but it possesses as a wide variety of ecosystems providing habitats for a diverse bird species. The key habitats of this island are shallow water marine habitats, including rocky and sandy inter-tidal habitats, offshore lagoons, rocky sub-tidal habitats, coral aggregations, sea grass beds and soft coral

habitats. Several lagoons and wetlands associated with mangrove and floodplain areas also occur on the island (Tomascik 1997). 
Field as well as literature surveys were conducted to collect data for this study. Seven field trips were conducted in April, 2016 and between October, 2016 and March, 17. However, due to frequent tropical storm and rough sea condition, study site could not be visited from May to September, 2016. A transect $(5.03 \mathrm{~L} \times 0.1 \mathrm{~W})$ line survey along the mid-section of the island from north to south and a shoreline survey was conducted for observing and recording the bird species (Fig. 1). The transect line surveys were conducted in the early morning (starting from the sunrise) and afternoon (starting from

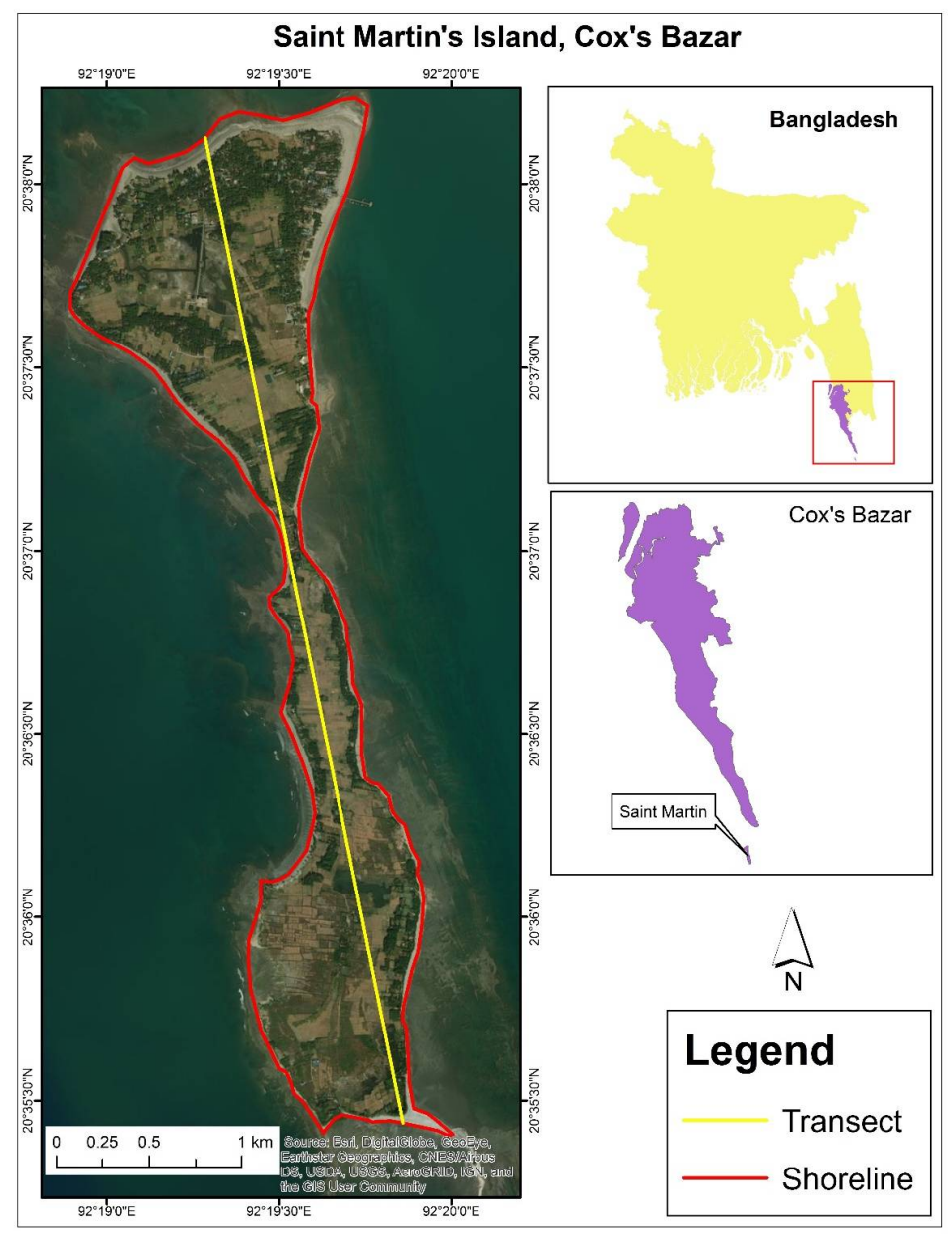

Fig. 1. Study site (St. Martin's Island of Bangladesh). The yellow line showing the transect line followed during the study from north to south of the island and the red line showing the shoreline survey area of the island. 
two and a half hours before the sunset). Shoreline survey was started two hours before the high tide and ended two hours after the high tide when they were distributed along the high water line as well as on the adjacent rocks. Relative abundance of bird species was measured into four categories: Very common (75 - 100\% encounter of the bird species during the total study period), common (50 - 75\%), uncommon (25-50\%) and rare (1 $25 \%$ ). Binocular (Ashika $10 \times 42$ ) and Telescope (Swarovski HD $20-60 \times 65 \mathrm{~mm}$ ) used to watch the birds in the field. Grimmett et al. (1999) and Chowdhury (2011) were used on the field to identify birds and photographs were taken whenever an identification problem arose in the field and subsequently consulted with expert birders. Besides, a thorough literature survey was conducted about previous bird records in the St. Martin's Island through available literature and reports (Islam 2001, Thompson and Islam 2010, BOBLME 2015).

\section{Results and Discussion}

A total of 112 species of birds belonging to 36 families under 15 orders were recorded from the St. Martin's Island (Table 1). Of these species, 37 (33\%) species were passerine and 75 (67\%) were non-passerine (Fig. 2); 11 (9.82\%) species were very common; 12 $(10.72 \%)$ were common; nine $(8.04 \%)$ were uncommon and $80(71.43 \%)$ were rare (Fig. 3). Four species, namely Bar-tailed godwit (Limosa lapponica), Black-tailed godwit (Limosa limosa), Eurasian curlew (Numenius arquata) and Black-headed ibis (Threskiornis melanocephalus) are near threatened and one species Black-bellied tern (Sterna acuticauda) is endangered (IUCN Red List of Threatened Species 2017) whether Black-bellied tern (Sterna acuticauda) is critically endangered and Black-headed ibis (Threskiornis melanocephalus) is vulnerable (IUCN Bangladesh 2015).

Passerine species: The passerine group consisted of 37 species belonging to 16 families of birds. Maximum six species was found under the family Motacillidae, and the families like Passeridae, Pycnonotidae, Alaudidae, Campephagidae and Ploceidae included one species each. However, more than 300 species of birds in Bangladesh are passerines (IUCN Bangladesh 2015).

Non-passerine species: Of the 112 bird species of this checklist, 75 species were nonpasserine. The order Charadriiformes dominated (34 species) among the non-passerines. The order Bucerotiformes, Caprimulgiformes, Suliformes and Pelecaniformes included one species each. Of all families of the recorded bird species, Scolopacidae contains large number of bird species (16 species). In Bangladesh, the orders Charadriiformes and Accipitriformes have higher numbers of species among the non-passerine birds (IUCN 
Table 1. Avifaunal checklist of the St. Martin's Island of Bangladesh.

\begin{tabular}{|c|c|c|c|c|c|}
\hline $\begin{array}{l}\text { Sl. } \\
\text { No. }\end{array}$ & Order & Family & English name & Scientific name & $\begin{array}{l}\text { Global } \\
\text { (National) } \\
\text { IUCN status }\end{array}$ \\
\hline 1 & Anseriformes & Anatidae & Northern shoveler* & Spatula clypeata & $\mathrm{LC}(\mathrm{LC})$ \\
\hline 2 & Anseriformes & Anatidae & Eurasian wigeon* & Marecapenelope & LC (LC) \\
\hline 3 & Anseriformes & Anatidae & Ruddy shelduck* & Tadornaferruginea & $\mathrm{LC}(\mathrm{LC})$ \\
\hline 4 & Anseriformes & Anatidae & Common shelduck* & Tadornatadorna & $\mathrm{LC}(\mathrm{LC})$ \\
\hline 5 & Anseriformes & Ardeidae & Indian pond heron & Ardeolagrayii & $\mathrm{LC}(\mathrm{LC})$ \\
\hline 6 & Anseriformes & Ardeidae & Grey heron* & Ardeacinerea & LC (LC) \\
\hline 7 & Anseriformes & Ardeidae & Little egret & Egrettagarzetta & $\mathrm{LC}(\mathrm{LC})$ \\
\hline 8 & Anseriformes & Ardeidae & Intermediate egret & Ardea intermedia & $\mathrm{LC}(\mathrm{LC})$ \\
\hline 9 & Anseriformes & Ardeidae & Cattle egret* & Bubulcus ibis & $\mathrm{LC}(\mathrm{LC})$ \\
\hline 10 & Anseriformes & Ardeidae & Striated heron* & Butoridesstiatus & $\mathrm{LC}(\mathrm{LC})$ \\
\hline 11 & Anseriformes & Ardeidae & Great egret* & Ardea alba & $\mathrm{LC}(\mathrm{LC})$ \\
\hline 12 & Anseriformes & Ardeidae & Pacific reef egret* & Egretta sacra & $\mathrm{LC}$ \\
\hline 13 & Anseriformes & Ardeidae & $\begin{array}{l}\text { Black-crowned night } \\
\text { Heron* }\end{array}$ & Nycticoraxnycticorax & $\mathrm{LC}(\mathrm{LC})$ \\
\hline 14 & Passeriformes & Passeridae & House sparrow & Passer domesticus & $\mathrm{LC}(\mathrm{LC})$ \\
\hline 15 & Passeriformes & Corvidae & House crow & Corvussplendens & $\mathrm{LC}(\mathrm{LC})$ \\
\hline 16 & Passeriformes & Corvidae & Large-billed crow & Corvusmacrorhynchos & $\mathrm{LC}(\mathrm{LC})$ \\
\hline 17 & Passeriformes & Dicruridae & Black drongo & Dicrurusmacrocercus & $\mathrm{LC}(\mathrm{LC})$ \\
\hline 18 & Passeriformes & Dicruridae & Ashy drongo* & Dicrurusleucophaeus & $\mathrm{LC}(\mathrm{LC})$ \\
\hline 19 & Passeriformes & Sturnidae & Common myna & Acridotherestristis & $\mathrm{LC}(\mathrm{LC})$ \\
\hline 20 & Passeriformes & Sturnidae & Asian pied starling & Gracupica contra & $\mathrm{LC}(\mathrm{LC})$ \\
\hline 21 & Passeriformes & Sturnidae & Asian glossy starling* & Aplonispanayensis & $\mathrm{LC}(\mathrm{LC})$ \\
\hline 22 & Passeriformes & Sturnidae & Rosy starling* & Pastor roseus & $\mathrm{LC}(\mathrm{LC})$ \\
\hline 23 & Passeriformes & Pycnonotidae & Red-vented bulbul & Pycnonotuscafer & $\mathrm{LC}(\mathrm{LC})$ \\
\hline 24 & Passeriformes & Alaudidae & Oriental skylark* & Alaudagulgula & $\mathrm{LC}(\mathrm{LC})$ \\
\hline 25 & Passeriformes & Campephagidae & $\begin{array}{l}\text { Black-winged } \\
\text { Cuckooshrike* }\end{array}$ & Lalage melaschistos & $\mathrm{LC}(\mathrm{LC})$ \\
\hline 26 & Passeriformes & Lanidae & Brown shrike & Laniuscristatus & $\mathrm{LC}(\mathrm{LC})$ \\
\hline 27 & Passeriformes & Lanidae & Grey-backed shrike* & Laniustephronotus & $\mathrm{LC}(\mathrm{LC})$ \\
\hline 28 & Passeriformes & Lanidae & Long-tailed shrike & Laniusschach & $\mathrm{LC}(\mathrm{LC})$ \\
\hline 29 & Passeriformes & Ploceidae & Baya weaver & Ploceusphilippinus & $\mathrm{LC}(\mathrm{LC})$ \\
\hline 30 & Passeriformes & Motacillidae & White wagtail & Motacilla alba & $\mathrm{LC}(\mathrm{LC})$ \\
\hline 33 & Passeriformes & Motacillidae & $\begin{array}{l}\text { Western yellow } \\
\text { Wagtail* }\end{array}$ & Motacilla flava & $\mathrm{LC}(\mathrm{LC})$ \\
\hline
\end{tabular}




\begin{tabular}{|c|c|c|c|c|c|}
\hline 34 & Passeriformes & Motacillidae & Paddyfield pipit & Anthusrufulus & LC (LC) \\
\hline 35 & Passeriformes & Motacillidae & Olive-backed pipit* & Anthushodgsoni & $\mathrm{LC}(\mathrm{LC})$ \\
\hline 36 & Passeriformes & Muscicapidae & Oriental magpie robin & Copsychussaularis & $\mathrm{LC}(\mathrm{LC})$ \\
\hline 37 & Passeriformes & Muscicapidae & Blue rock thrush & Monticola solitarius & $\mathrm{LC}(\mathrm{LC})$ \\
\hline 38 & Passeriformes & Muscicapidae & Taiga flycatcher & Ficedulaalbicilla & LC (LC) \\
\hline 39 & Passeriformes & Estrillidae & Scaly-breasted munia & Lonchurapunctulata & LC (LC) \\
\hline 40 & Passeriformes & Estrillidae & Black-headed munia & Lonchuramalacca & LC (LC) \\
\hline 41 & Passeriformes & Nectarinidae & Purple-rumped sunbird & Leptocomazeylonica & LC (LC) \\
\hline 42 & Passeriformes & Nectarinidae & Purple sunbird & Cinnyrisasiaticus & LC (LC) \\
\hline 43 & Passeriformes & Sylviidae & Common chiffchaff & Phylloscopuscollybita & LC (LC) \\
\hline 44 & Passeriformes & Sylviidae & Common tailorbird & Orthotomussutorius & LC (LC) \\
\hline 45 & Passeriformes & Sylviidae & Thick-billed warbler & Acrocephalusaedon & LC (LC) \\
\hline 46 & Passeriformes & Hirundinidae & Barn swallow & Hirundorustica & $\mathrm{LC}(\mathrm{LC})$ \\
\hline 47 & Passeriformes & Hirundinidae & Red-rumped swallow* & Hirundodaurica & $\mathrm{LC}(\mathrm{LC})$ \\
\hline 48 & Passeriformes & Oriolidae & Black-naped oriole* & Orioluschinensis & LC (LC) \\
\hline 49 & Passeriformes & Oriolidae & Black-hooded oriole* & Oriolusxanthornus & LC (LC) \\
\hline 50 & Columbiformes & Columbidae & Eurasian golden oriole & Oriolusoriolus & $\mathrm{LC}(\mathrm{LC})$ \\
\hline 51 & Columbiformes & Columbidae & Rock pigeon & Columba livia & $\mathrm{LC}(\mathrm{LC})$ \\
\hline 52 & Columbiformes & Columbidae & Spotted dove & Streptopeliasuratensis & LC (LC) \\
\hline 53 & Columbiformes & Columbidae & Eurasian collared dove & Streptopeliadecaocto & LC (LC) \\
\hline 54 & Piciformes & Picidae & Eurasian wryneck & Jynxtorquilla & LC (LC) \\
\hline 55 & Piciformes & Picidae & $\begin{array}{l}\text { Lesser golden-backed } \\
\text { Woodpecker* }\end{array}$ & Dinopiumbenghalense & LC (LC) \\
\hline 56 & Piciformes & Picidae & Fulvous breasted & $\begin{array}{l}\text { Dendrocoposmacei } \\
\text { Woodpecker }\end{array}$ & LC (LC) \\
\hline 57 & Coraciformes & Alcedinidae & Common kingfisher & Alcedoatthis & $\mathrm{LC}(\mathrm{LC})$ \\
\hline 58 & Coraciformes & Alcedinidae & $\begin{array}{l}\text { White-throated } \\
\text { Kingfisher }\end{array}$ & Halcyon smyrnensis & $\mathrm{LC}(\mathrm{LC})$ \\
\hline 59 & Coraciformes & Alcedinidae & $\begin{array}{l}\text { Black-capped } \\
\text { Kingfisher* }\end{array}$ & Halcyon pileata & LC (LC) \\
\hline 60 & Coraciformes & Alcedinidae & Collared kingfisher* & Todiramphuschloris & $\mathrm{LC}(\mathrm{LC})$ \\
\hline 61 & Coraciformes & Meropidae & Green bee-eater* & Meropsorientalis & $\mathrm{LC}(\mathrm{LC})$ \\
\hline 62 & Coraciformes & Meropidae & $\begin{array}{l}\text { Chestnut-headed bee- } \\
\text { Eater* }\end{array}$ & Merops leschenaultia & $\mathrm{LC}(\mathrm{LC})$ \\
\hline 63 & Cuculiformes & Cuculidae & Plaintive cuckoo* & Cacomantismerulinus & $\mathrm{LC}(\mathrm{LC})$ \\
\hline 64 & Cuculiformes & Cuculidae & Asian koel & Eudynamysscolopaceus & $\mathrm{LC}(\mathrm{LC})$ \\
\hline 65 & Cuculiformes & Cuculidae & Greater coucal & Centropussinensis & $\mathrm{LC}(\mathrm{LC})$ \\
\hline 66 & Strigiformes & Tytonidae & Spotted owlet* & Athene drama & $\mathrm{LC}(\mathrm{LC})$ \\
\hline
\end{tabular}




\begin{tabular}{|c|c|c|c|c|c|}
\hline 67 & Strigiformes & Tytonidae & Common barn owl* & Tyto alba & $\mathrm{LC}(\mathrm{LC})$ \\
\hline 68 & Bucerotiformes & Upupidae & Common hoopoe & Upupaepops & LC (LC) \\
\hline 69 & Caprimulgiformes & Hemiprocnidae & Asian palm swift & Cypsiurusbalasiensis & LC (LC) \\
\hline 70 & Gruiformes & Rallidae & Ruddy breasted crake & Zaporniafusca & LC (LC) \\
\hline 71 & Gruiformes & Rallidae & Common moorehen* & Gallinulachloropus & $\mathrm{LC}(\mathrm{LC})$ \\
\hline 72 & Charadriiformes & Scolopacidae & Common snipe & Gallinagogallinago & $\mathrm{LC}(\mathrm{LC})$ \\
\hline 73 & Charadriiformes & Scolopacidae & Common sandpiper & Actitishypoleucos & $\mathrm{LC}(\mathrm{LC})$ \\
\hline 74 & Charadriiformes & Scolopacidae & Eurasian curlew & Numeniusarquata & NT (NT) \\
\hline 75 & Charadriiformes & Scolopacidae & Whimbrel & Numeniusphaeopus & LC (LC) \\
\hline 76 & Charadriiformes & Scolopacidae & Ruddy turnstone & Arenariainterpres & LC (LC) \\
\hline 77 & Charadriiformes & Scolopacidae & Ruff & Calidrispugnax & $\mathrm{LC}(\mathrm{LC})$ \\
\hline 78 & Charadriiformes & Scolopacidae & Sanderling* & Calidris alba & LC (LC) \\
\hline 79 & Charadriiformes & Scolopacidae & Pintail snipe* & Gallinagostenura & LC (LC) \\
\hline 80 & Charadriiformes & Scolopacidae & Broad-billed sandpiper* & Limicolafalcinellus & $\mathrm{LC}(\mathrm{LC})$ \\
\hline 81 & Charadriiformes & Scolopacidae & Bar-tailed godwit* & Limosalapponica & NT (NT) \\
\hline 82 & Charadriiformes & Scolopacidae & Black-tailed godwit* & Limosalimosa & NT (NT) \\
\hline 83 & Charadriiformes & Scolopacidae & Spotted redshank* & Tringaerythropus & $\mathrm{LC}(\mathrm{LC})$ \\
\hline 84 & Charadriiformes & Scolopacidae & Wood sandpiper* & Tringaglareola & $\mathrm{LC}(\mathrm{LC})$ \\
\hline 85 & Charadriiformes & Scolopacidae & Common greenshank* & Tringanebularia & LC (LC) \\
\hline 86 & Charadriiformes & Scolopacidae & Common redshank* & Tringa tetanus & $\mathrm{LC}(\mathrm{LC})$ \\
\hline 87 & Charadriiformes & Scolopacidae & Terek sandpiper* & Xenuscinereus & $\mathrm{LC}(\mathrm{LC})$ \\
\hline 88 & Charadriiformes & Charadriidae & Lesser sand plover & Charadriusmongolus & LC (LC) \\
\hline 89 & Charadriiformes & Charadriidae & Greater Sand plover & $\begin{array}{l}\text { Charadrius } \\
\text { leschenaultia }\end{array}$ & $\mathrm{LC}(\mathrm{LC})$ \\
\hline 90 & Charadriiformes & Charadriidae & Pacific golden plover & Pluvialisfulva & LC (LC) \\
\hline 91 & Charadriiformes & Charadriidae & Little ringed plover & Charadriusdubius & $\mathrm{LC}(\mathrm{LC})$ \\
\hline 92 & Charadriiformes & Charadriidae & Grey-headed lapwing & Vanelluscinereus & $\mathrm{LC}(\mathrm{LC})$ \\
\hline 93 & Charadriiformes & Charadriidae & Kentish plover & $\begin{array}{l}\text { Charadrius } \\
\text { alexandrinus }\end{array}$ & LC (LC) \\
\hline 94 & Charadriiformes & Charadriidae & Grey plover* & Pluvialissquatarola & LC (LC) \\
\hline 95 & Charadriiformes & Charadriidae & Red-wattled lapwing* & Vanellus indicus & LC (LC) \\
\hline 96 & Charadriiformes & Laridae & Little tern & Sterna albifrons & $\mathrm{LC}(\mathrm{LC})$ \\
\hline 97 & Charadriiformes & Laridae & Black-headed gull & Larusridibundus & $\mathrm{LC}(\mathrm{LC})$ \\
\hline 98 & Charadriiformes & Laridae & Brown-headed gull & Larusbrunnicephalus & $\mathrm{LC}(\mathrm{LC})$ \\
\hline 99 & Charadriiformes & Laridae & Whiskered tern* & Chlidoniashybridus & LC (LC) \\
\hline 100 & Charadriiformes & Laridae & Gull-billed tern* & Gelochelidonnilotica & LC (LC) \\
\hline 101 & Charadriiformes & Laridae & Pallas's gull* & Larusichthyaetus & LC (LC) \\
\hline 102 & Charadriiformes & Laridae & Black-bellied tern* & Sterna acuticauda & EN (CR) \\
\hline
\end{tabular}




\begin{tabular}{|c|c|c|c|c|c|}
\hline 103 & Charadriiformes & Laridae & Lesser crested tern* & Sterna bergii & $\mathrm{LC}(\mathrm{LC})$ \\
\hline 104 & Charadriiformes & Laridae & Caspian tern* & Sterna caspia & $\mathrm{LC}(\mathrm{LC})$ \\
\hline 105 & Charadriiformes & Glareolidae & Oriental pratincole* & Glareolamaldivarum & $\mathrm{LC}(\mathrm{LC})$ \\
\hline 106 & Suliformes & Phalacrocoracidae & Little cormorant & Microcarboniger & $\mathrm{LC}(\mathrm{LC})$ \\
\hline 107 & Pelecaniformes & Threskiornithidae & Black-headed ibis & $\begin{array}{l}\text { Threskiornis } \\
\text { melanocephalus }\end{array}$ & NT (VU) \\
\hline 108 & Accipitriformes & Accipitridae & Shikra* & Accipiter badius & $\mathrm{LC}(\mathrm{LC})$ \\
\hline 109 & Accipitriformes & Accipitridae & Brahminy kite* & Haliastur Indus & LC (LC) \\
\hline 110 & Accipitriformes & Pandionidae & Osprey* & Pandion haliaetus & $\mathrm{LC}(\mathrm{LC})$ \\
\hline 111 & Falconiformes & Falconidae & Peregrine falcon & Falco peregrines & LC (LC) \\
\hline 112 & Falconiformes & Falconidae & Common kestrel* & Falco tinnunculus & $\mathrm{LC}(\mathrm{LC})$ \\
\hline
\end{tabular}

$(\mathrm{LC}=$ Least concern, $\mathrm{NT}=$ Near threatened, $\mathrm{VU}=$ Vulnerable, $\mathrm{CR}=$ Critically endangered and $\mathrm{EN}=$ Endangered). * Historic records

Passerine and Non--Passerine bird sp.

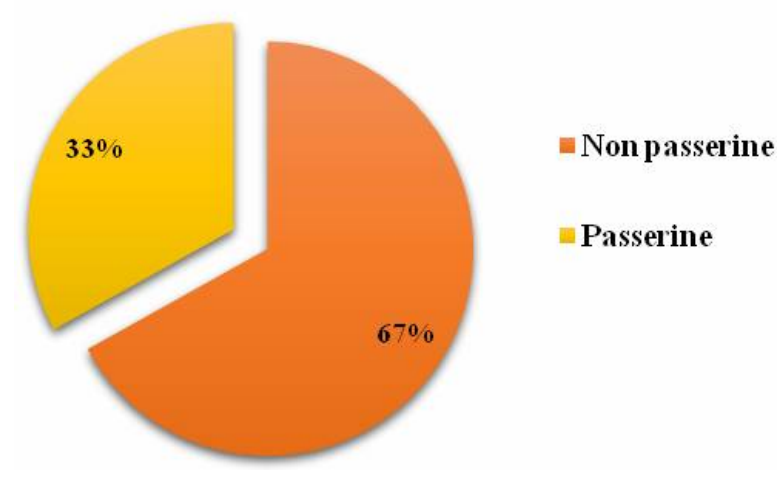

Fig. 2. Two major groups of the recorded bird species; non-passerine birds dominated in this landscape showing its ecological uniqueness.

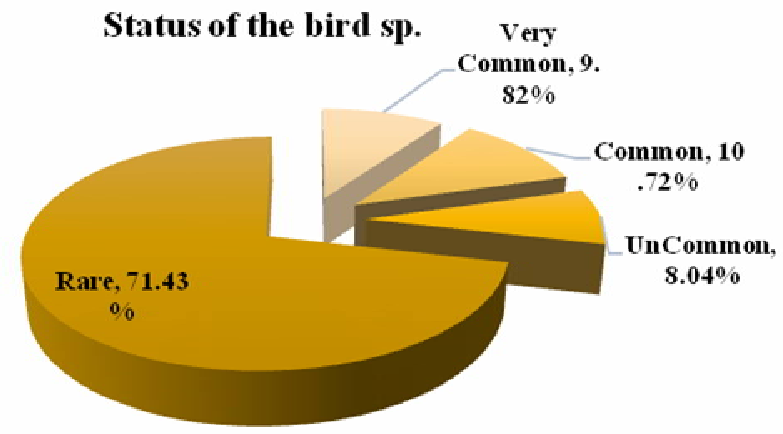

Fig. 3. Status of bird species at the St. Martin's Island; most bird species are rare and hence emphasizing the need of protection of this ecosystem. 
Bangladesh 2015). Ahsan and Hannan (2002) recorded 141 bird species at the Karnaphuli river delta and adjacent areas of Chittagong, Bangladesh. Comparing to Karnaphuli river delta and adjacent areas of Chittagong, The St. Martin's Island is a very small Island, but 112 bird species are recorded. A total of 28 wader species was recorded at the Sonadia Island $\left(49.16 \mathrm{~km}^{2}\right.$ ), Cox's Bazar, south east coast of Bangladesh (Chowdhury et al. 2011). In contrast, 24 species of waders were found in the St. Martin's Island of Bangladesh. However, the scale of congregation differs in a large scale. Sonadia Island recorded a congregation of 12,710 individuals of shorebirds (Chowdhury et al. 2011) compared to the St. Martin's Island where a congregation of 820 individuals had been recorded.

We hope that this checklist will act as a baseline source for further study on avifaunal species and their conservation at this site in the face of growing human activities and interventions. Also it will help in future monitoring the environmental health of this island.

\section{Acknowledgements}

The study was supported by the Jagannath University Research Grant (2015-2016) and Research Fellowship from the National Science and Technology of Bangladesh (2016). The authors are thankful to Ataur Rahman and his family for providing support on field. They are deeply grateful to Md. Salauddin, Department of Geography \& Environment Science, Jagannath University, Dhaka, Bangladesh for creating the map of the study site using ArcGIS software.

\section{References}

Ahsan, M.F. and K. Khanom. 2005. Birds of the Chittagong University Campus, Chittagong. The Chittagong Univ. J. Sci. 29(1): 77-88.

Ahsan, M.F. and M.A. Hannan. 2002. Birds of the Karnaphuli river delta and adjacent areas in Chittagong, Bangladesh. Zoos' Print Journal 17(9): 875-882.

BOBLME, 2015. Saint Martin's biological survey report, Bangladesh. BOBLME-2015-Ecology48.

Bryan, K., T. Gallucci, G. Lasley, M. Lockwood, and D.H. Riskind. 2006. A checklist of Texas birds. PWD BK P4000-000M (4/06). ISBN: 1-885696-09-4

Chowdhury, S.U. 2011. A Pictorial Field Guide to the Shorebirds of Bangladesh. Dhaka, Bangladesh. 59 pp.

Chowdhury, S.U. 2016. The Daily Star (Bangladesh). Website: file://F:/Literature\%20surveys/ FEATURE_\%20Nature\%20Quest \%20-\%20Blue\%20beauty\%20of\%20Saint\%20Martin's\% 20 _\%20Asia\%20News\%20Network.html. [Accessed on 31 January 2018.] 
Chowdhury, S.U., M. Foysal, D.K. Das, S. Mohsanin, M.A.A. Diyan, and A.B.M.S. Alam. 2011. Seasonal occurrence and site use by shorebirds at Sonadia Island, Cox's Bazar, Bangladesh. Wader Study Group Bull. 118(2): 77-81.

DoE, 2015. The Fifth National Report of Bangladesh to the Convention on Biological Diversity. Department of Environment, Ministry of Environment and Forests, Government of the People's Republic of Bangladesh, Dhaka. 125 pp.

Grimmett, R., C. Inskipp, and T. Inskipp. 1999. Pocket Guide to the Birds of the INDIAN Subcontinent. Oxford University Press, YMCA Library Building, Jai Singh Road, New Delhi 110001.384 pp.

Hossain, M.A., M.S.E. Mahfuj, S.M.A. Rashid, M.I. Miah, and M.N. Ahsan. 2013. Present status of conservation and management of sea turtle in Cox's Bazar district, Bangladesh. Mesopot. J. Mar. Sci. 28(1): 45-60.

Islam, M.S. 2001. Sea Level Changes in Bangladesh: The Last Ten Thousand Years. Asiatic Soc. Bangladesh. Dhaka. 185 pp.

IUCN Bangladesh 2015. Red List of Bangladesh Volume 3: Birds. IUCN, International Union for Conservation of Nature, Bangladesh Country Office, Dhaka, Bangladesh. pp. i-xvi, 1-676.

Khan, M.M.H. 2008. Protected Areas of Bangladesh- A Guide to Wildlife Nishorgo Program, Bangladesh Forest Department, Dhaka, Bangladesh. 304 pp.

Rashid, S.M.A. and Z.M. Islam, 2005. Research and Conservation of Marine Turtles in Bangladesh. In: Marine Turtles of Indian subcontinent (ed. Kartik Shankar, B. C. Choudhury). pp. 200-216, Universities press. California.

The Daily Star (Bangladesh). 2010. Website: http://www.thedailystar.net/news-detail-122463. [Accessed on 31 January 2018.]

The IUCN Red List of Threatened Species. 2017. Version 2017-3.Website: http://www. iucnredlist.org/. [Accessed on 31 January 2018.]

Thompson, P.M. and M.A. Islam, (Eds.). 2010. Environmental Profile of St. Martin's Island, CWBMP, United Nations Development Programme, Dhaka. $151 \mathrm{pp}$.

Tomascik, T. 1997. Management Plan for Coral Resources of NarikelJinjira (St. Martin's Island). Final Report. National Conservation Strategy Implementation Project-1, Dhaka, Bangladesh. $126 \mathrm{pp}$.

Zöckler, C. 2005. Migratory bird species as indicators for the State of the environment. Huntingdon Road Cambridge, UK. Biodiversity 6(3): 219.

(Revised copy received on 23.9.2018) 